\title{
Matthias Hild*
}

\section{Fair Kidney Allocation Based on Waiting Time}

\begin{abstract}
We study the allocation of cadaveric donor kidneys for transplantation based merely on waiting time. This simple allocation rule turns out to possess very attractive ethical and medical properties. Current allocation rules, on the other hand, violate some basic requirements of distributive justice. Perhaps for fear of exacerbating these problems, these rules also fail to consider criteria such as sex, age and race although certain combinations of these criteria are known to affect graft survival rates. We demonstrate that allocation by waiting time automatically protects disadvantaged patient types and puts them in a near to optimal position. The inclusion of sex, age and race will therefore not lead to morally unacceptable allocations. This allows individual patients to improve the expected survival time of their graft relative to the status quo without being penalized by the allocation rule. Moreover, decisions about when to start compromising on expected graft survival rates in favour of shorter waiting times are made locally by patients and their medical advisers rather than by a centralized protocol.
\end{abstract}

\section{Introduction}

We propose to allocate cadaveric donor kidneys primarily on the basis of recipients' waiting time. When a new donor organ becomes available, the patient with the longest waiting time is first offered this organ for transplantation. If the patient and her medical advisers reject the organ, it is offered to the next patient on the waiting list who can again accept the organ or pass it on to the next patient etc. Refusal to accept an organ does not alter patients' position on the waiting list. We provide an explicit protocol for this process. We retain some control over the patients' range of choices to exclude medically impossible allocations (e.g., incompatible blood types) and to guarantee that the patient is in a fit state to undergo transplantation. Waiting time is measured from the start of dialysis and ties in waiting time are broken randomly. ${ }^{1}$ Some modifications will be made to accommodate the special needs of children or patients with high medical urgency. These patient groups are already recognized by the current allocation rules to be significantly different from other patients and to deserve special considerations. Criteria that equalize regional or international export/import balances can also be added if we wish.

Allocation by waiting time (AWT) is a simple, decentralized and transparent mechanism that is responsive to the patients' and their medical advisers' judge-

* I wish to thank Bill for his thoughtful comments.

1 We could also consider measuring waiting time from the time of the patient's enlistment. 
ment about how to balance waiting time against other (medical) criteria. We show that AWT removes the tension that is present in current allocation rules between optimal medical predictors of graft survival and concerns of distributive justice. We shall show that AWT automatically protects disadvantaged patient types. For example, it leads to an automatic protection of patients with blood type $\mathrm{O}$. Moreover, it allows patients to improve the expected survival time of their graft relative to the status quo without being penalized by the allocation system. These optimality properties are established by a simple statistical argument: Patients of a rare medical type, for example, wait on average longer before an organ of their rare type becomes available. This increased waiting time gives rare patients automatically a higher priority under AWT. Hence, the probability is very small that an organ of a rare type will be allocated to a patient of a more common type.

The allocation methods currently used in Europe and North America already give a significant weight to a patient's waiting time. Wujciak/Opelz (1993) made an impressive argument for using waiting time as a criterion in addition to HLA matches and PRA sensitization (measures of antigen and antibody compatibility). In the current allocation rules of Eurotransplant and the American United Network for Organ Sharing (UNOS), HLA matching is traded off against long waiting times so that patients of rare medical types can become eligible for an organ with a compromised HLA match. For short waiting times, patients of rare medical types are at a clear disadvantage because they are unlikely to find an organ with a good HLA match. This disadvantage is only partly offset by the inclusion of 'matchbility' (the probability of finding a better match in the future) as a criterion. Rare patient types are forced to compete for organs mainly through prolonged waiting times. They are not given the opportunity to determine their own trade-off between additional waiting time and a compromised HLA match. In North America, this leads to imbalances in the racial composition of the recipients. Moreover, morally sensitive patient attributes such as sex, age and race are not systematically taken into account by the current allocation practice although some of their combinations appear to be correlated with graft survival rates (Persijn/Smits/De Meester 1999). If these criteria were taken into account, additional imbalances would arise. In Europe, for instance, the 'old-for-old' programme in which kidneys from older donors are allocated to older recipients meets with some resistance on the part of older patients. Such ethical problems can only be expected to intensify as non-immunological factors continue to emerge as predictors of graft survival rates.

It is clear that any allocation method must make certain trade-offs between medical criteria, waiting time and other special attributes of the patients. AWT determines these trade-offs locally through the decisions of patients and their medical advisers. Currently used allocation methods specify such trade-offs in a quantitative manner through a centralized allocation protocol. Patients collect a certain number of points, for instance, for medical criteria (HLA matching, PRA sensitization, matchability) and for waiting time. The relative number of points awarded reflects the trade-offs that are implicit in the allocation method. This point system has to be adjusted frequently to respond to medical innovation and 
unfulfilled concerns for distributive justice. In economic theory it is well-known that such trade-offs are notoriously difficult, if not impossible, to make for a central decision maker like Eurotransplant or UNOS. More importantly, there is no sound medical or moral basis for making these trade-offs on behalf of the patients. Patients differ strongly in their psychological, social and emotional condition. They may even differ in their rational evaluation of the risks and the benefits of dialysis and transplantation.

Under the current system, patients are in a very poor position to choose their own trade-offs. Firstly, the system structurally penalizes selective patients who want to achieve better medical matches than chosen by the central allocation protocol. For instance, patients who bypass an offer because they want to match the donor organ for sex or age are not guaranteed to be offered the next available organ. Instead, they will have to compete for the next organ with patients who will have newly joined the waiting list and who may have a better medical compatibility with the next organ. In other words, if a selective patient turns down an organ, then a weaker competitor receives the organ and is removed from the waiting list while new and stronger competitors may still join the waiting list. A selective patient's ability to choose is therefore not preserved from round to round. Secondly, patients cannot achieve shorter waiting times than chosen by the central allocation protocol even if they are prepared to accept marginal organs. To use a technical term from welfare theory, the current allocation system is not Pareto optimal or efficient (cf. below). Thirdly, there is no explicit protocol to accommodate the patients' choices in the allocation process. Fourthly, patients lack relevant medical information. A project is currently under way to design a decision support system that conveys medically relevant information to the patient and their medical advisers in a cognitively and psychologically tractable format. When joining the waiting list, we envisage the patients and their medical advisers to draw up a plan that specifies at what time they wish to accept an organ with specific characteristics. For instance, a patient may decide to accept a 'marginal' organ (an organ of poor quality) in return for a short waiting time but not after a long waiting time. Patients have the opportunity to revise this plan on a regular basis. Patients can also choose from a set of default plans or recommendations by the central allocation authority. Patients may prefer to delegate the choice of such a plan to their medical advisers. Transplant surgeons will always play an important role in this process because of unquantifiable characteristics of donor organs that require a surgeon's medical judgement.

Although high graft survival rates are not the only relevant criterion by which to judge an allocation mechanism, they are certainly one very important criterion. The average medical optimality of AWT depends on local decisions and local evaluations of acceptable trade-offs. Patients have a definite incentive to maximize their graft survival rates, especially in view of medical side effects and complications arising from poor compatibility. Moreover, AWT allows the systematic inclusion of medical criteria such as age, sex and race that provide improved predictors of a graft's expected survival time. This may suggest that the medical performance of AWT will compare favourably with that of the cur- 
rent allocation rules because it taps into a potential for improvement. The exact performance of AWT can only be gauged once we have collected data on patients' trade-offs.

\section{Evaluation}

\subsection{Optimal Compensation of Disadvantage}

AWT promotes equality of opportunity among patients. Equality of opportunity does not imply that all patients receive an organ with the same expected survival time after the same waiting period. The persistent shortage of suitable donor organs and the medical differences among patients make it impossible to achieve such equality of outcomes. Equality of opportunity implies, however, that the effect of any natural disadvantage is kept as small as possible. AWT does indeed possess this optimality property. It ensures that patients of a disadvantaged medical type wait for a time that is close to the minimum possible waiting time. Any remaining imbalances are either the result of a natural disadvantage for which our current medical technology is unable to compensate or the result of the disadvantaged patient's personal choice. Since AWT automatically protects disadvantaged patients, special precautions taken on behalf of these patients are superfluous. We will examine these optimality properties from a statistical viewpoint. Any allocation rule has to operate in a stochastic environment in which donor organs and new patients appear in a random order and at random times and in which the survival time of a graft can only be predicted with a certain probability. No algorithm can avoid inequalities that are caused by such random variations in the supply and demand for organs and in the actual survival time of a graft. The best we can do is to compare alternative allocation rules on a statistical basis. ${ }^{2}$

Over and above providing equality of opportunity, AWT has additional, morally desirable properties that concern the manner in which it allocates scarce resources among patients that are not distinguished by natural advantages or disadvantages. Firstly, AWT results in allocations in which we cannot make any individual better off without making some individual worse off. ${ }^{3}$ For obvious reasons, such allocations are also called 'efficient' or Pareto optimal (named after the Italian economist Vilfredo Pareto, 1848-1923). Since both the Eurotransplant and the UNOS rule do not reflect patients' preferences, they threaten to produce allocations that could be improved without making any patient worse off. Secondly, AWT results in 'envy-free' allocations in which no patient would want to switch places with anybody else who joined the waiting list at roughly

2 Hild (2001a; 2001b) provides theoretical reasons why such an ex ante evaluation is preferable to an ex post analysis in terms of Quality Adjusted Life Years.

3 We resolve a patient's indifference between accepting and rejecting an organ by passing the organ on to the next patient. We assume that if some patient is indifferent between accepting and rejecting an organ, there is some other patient with a shorter waiting time who prefers to accept the organ. This guarantees a Pareto optimal allocation of the chance to receive an organ depending on waiting time. 
the same time (Tinbergen 1953, Foley 1967, Thomson/Varian 1985). In other words, no patient $i$ would prefer to receive an organ allocated to another patient $j$ if $i$ also had to accept the same expected waiting time for this organ as patient $j$ did (assuming that ceteris paribus patients prefer shorter waiting times). In this sense, envy-free allocations provide a conflict-free and harmonious state of affairs. As a sorry implication of the current donation rates, this condition is only satisfied for patients who join the waiting list at roughly the same time. Since donor organs currently arrive at a slower rate than patients, the waiting list must be increasing in size and future patients will on average have to wait longer than current patients. If donation rates were one day to exceed kidney failure rates, the situation would conversely favour patients who are born later and join the waiting list at a later time. In the Eurotransplant and UNOS algorithms, waiting time gathers an increasing weight and will require repeated adjustments. We will later consider a formula for compensating for this temporal effect.

\subsection{Rare Medical Types}

It is easy to see why AWT puts disadvantaged patients in a near optimal position. As a first example, we focus on disadvantages that are linked to rare medical types. Without any loss of generality, we may suppose that we have patients and organs of two types, the first representing a common combination of medical characteristics and the second representing a rare combination. AWT guarantees that with high probability an organ of a rare medical type is not misallocated to a patient of a common medical type. The reason is simply that patients of a rare medical type on average wait longer until the next organ of their type becomes available. On average, this increases their waiting time and moves them closer to the front of the waiting list. The rare patient type is therefore automatically eligible for the next organ of the rare organ type.

This intuitive analysis is borne out by a precise statistical treatment (cf. Appendix 187). The probability of misallocating an organ of a rare type to a patient of a common type quickly falls to zero as the number of allocated organs increases. If a rare type occurs with a frequency of $5 \%$ in the population of donors and recipients, the probability of a misallocation falls to $1 \%$ as we go through less than 200 allocations. Even in the European allocation system which has a smaller volume of allocations than its American counterpart, 200 organs become available in less than 4 weeks. Figure 1 charts this probability of a misallocation for different frequencies of the 'rare' type. The lower part of this figure shows a contour chart of this probability surface. For any frequency, the fallibility of AWT is reduced to below $1 \%$ after as few as 800 allocations. It is by no means necessary to assume that the frequency of the rare type be the same in the population of donors and in the population of recipients. Our precise calculations let us make predictions about situations where these frequencies differ. The probability of a misallocation is, of course, not the only relevant measure of optimality. Another measure of allocative efficiency is the average proportion of misallocated organs of the rare type. This measure is not plotted here but, displaying the same behaviour that we saw before, it quickly falls to 

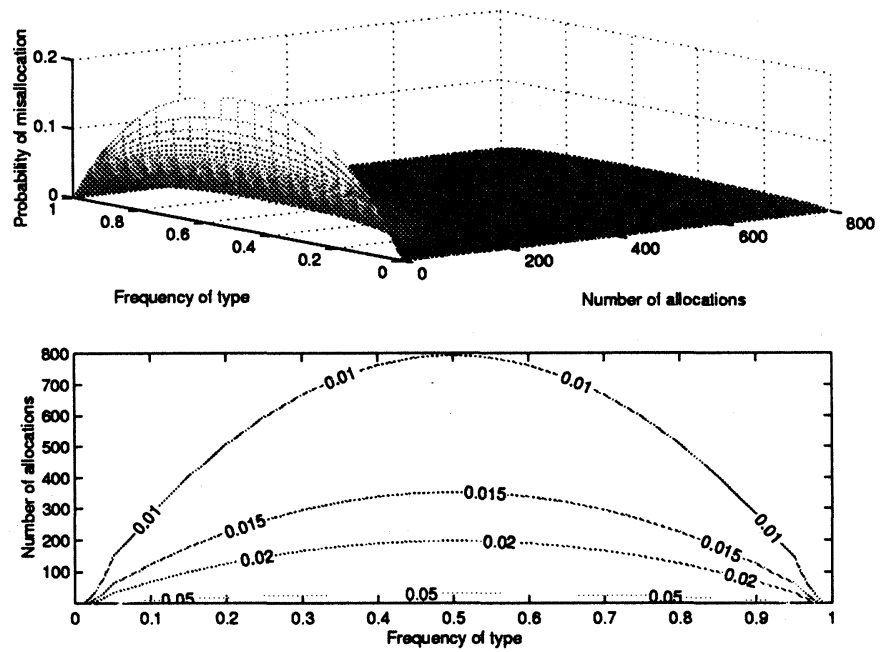

Figure 1: Probability of misallocation.

$$
(\alpha=1)
$$

zero as we go through a year's supply of donor organs. ${ }^{4}$

In the USA, the focus on medical criteria (HLA matching, PRA sensitization) disadvantages less frequent population groups, particularly African-Americans who wait significantly longer for kidney transplants than do Caucasian patients. Leffell/Zachary (1999) conclude that the 1995 policy change in the UNOS allocation rule did not remedy this problem. Although Caucasian patients constitute only $51.2 \%$ of the total US patient pool, they receive $78.5 \%$ of all organs that are allocated with 0 HLA mismatches and $54.4 \%$ of all other organs even after the 1995 revisions of the allocation rule. While African-Americans constitute $30.6 \%$ of the total patient pool, they receive only $10.5 \%$ of all organs that are allocated with 0 HLA mismatches. This imbalance arises from the immunological characteristics of African-Americans and from the weight that is given to these characteristics. Racial imbalances are a troublesome development of a well-intentioned allocation rule which clearly shows that the authority to determine trade-offs and to compromise on HLA matching must be put back in the hands of the medically disadvantaged patients themselves. For a rare type with a frequency of $30 \%$ in the overall population, the probability of a misallocation falls to $1.5 \%$ after less than 300 allocations.

Our calculations are strictly based on a worst-case scenario. We assume that patients of a rare type can only accept organs of the same type and that all patients of the common type accept any organ that is offered to them. This extreme assumption simulates a situation of extreme competition and of an ex-

4 We note that a rare patient type will for statistical reasons suffer from a greater variation in the duration of waiting times. It is outside of our power to compensate the rare patient type for this statistical disadvantage. 
treme disadvantage of the rare type. Remarkably, AWT yields close to optimal results even in this extreme environment. Our second conservative assumption concerns the initial composition of the waiting list. Our calculations assume that the waiting list has no history and is randomly composed of rare and common types. In reality, we inherit a waiting list that already contains patients of the rare type at the front of the waiting list. This decreases the probability of a misallocation even further. Our calculations also allow us to study an optimal initial configuration of the waiting list before we implement AWT. In other words, a fine-tuned implementation of AWT can decrease the probability of a misallocation even further. As a singular measure, fine-tuning involves moving some disadvantaged patients further to the front of the waiting list and then letting AWT work on its own. A third conservative assumption concerns the proportion $\alpha$ of those rare patients who choose to maximize their medical outcome regardless of other factors such as waiting time. Suppose, for example, rare patients occur with a frequency of $30 \%$ in the population. Suppose, furthermore, that $50 \%$ of these rare patients are selective and accept only an organ of the rare type. In this case, the selective rare patients can fairly demand $15 \%$ of all organs of the rare type. Figure 2 shows that the selective group of rare patients does indeed receive almost exactly this amount of rare organs. This figure plots the difference between the actual and the minimal rate of allocation of rare organs to common patients. This difference falls quickly to zero. Hence, patients of the rare type will not accumulate on the waiting list since they receive in the long run exactly their share of rare organs. Figure 3 plots the same distance for a disadvantage patient type that occurs with a frequency of $50 \%$ in the population. Finally, we note that our calculations indulge in one simplifying assumption. They assume that patients do not rejoin the waiting list for a second transplant. In reality, Eurotransplant reported $18 \%$ of second time transplantations among cadaveric transplants for 1999. This simplifying assumption allows us to derive an analytic description of our problem while we would otherwise have to resort to simulations.

\subsection{Asymmetric Medical Types}

Disadvantages can also arise from asymmetries in the capability of a medical type to donate and to receive organs. Some medical types can donate organs to patients of other types without affecting graft survival rates, while recipients of this type maximize their graft survival rates only when they receive an organ from a donor of same type. Organs of blood type $\mathrm{O}$ can be allocated patients of any blood type but patients of type $O$ can only receive organs of type $O$. Both the Eurotransplant and the UNOS rule make special provisions for patients of blood type $\mathrm{O}$. The same statistical argument as above establishes that AWT automatically protects patients of blood type $\mathrm{O}$ and that no additional protection mechanisms are required. In 1999, Eurotransplant reported that the proportion of patients of blood type $\mathrm{O}$ was $39 \%$ among patients newly added to the waiting list and $48 \%$ among all patients on the waiting list while $44 \%$ of all donors were of this type. In the same year, UNOS reports for the USA that $53 \%$ of all 


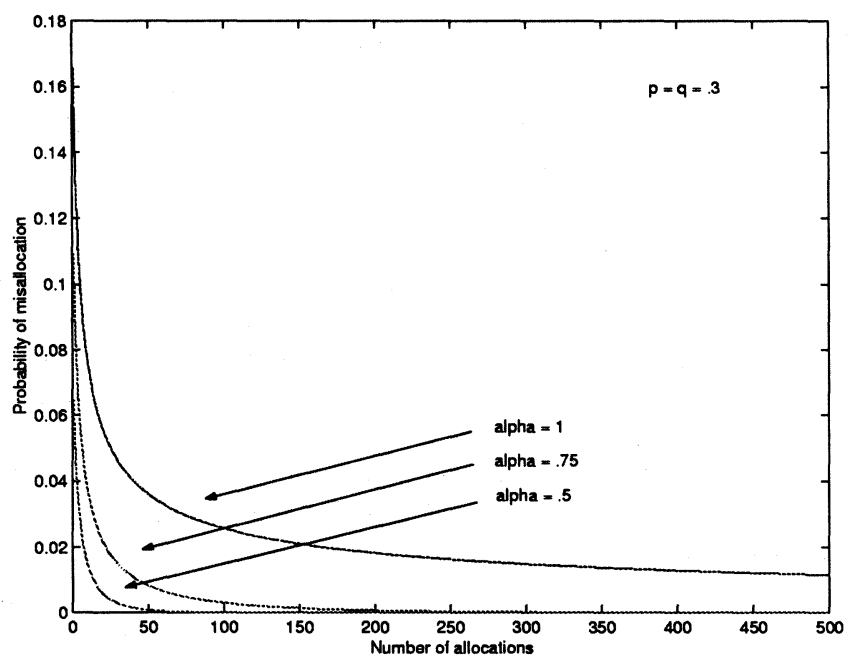

Figure 2: Distance from optimum for different $\alpha$. Frequency of disadvantaged patient type $=.3$.



Figure 3: Distance from optimum for different $\alpha$. Frequency of disadvantaged patient type $=.5$. 


\begin{tabular}{|c||c|c|}
\hline & number of transplants & 3 year survival rate \\
\hline \hline male to female & 1771 & $74 \%$ \\
male to male & 3109 & $76 \%$ \\
female to female & 765 & $74 \%$ \\
female to male & 1244 & $70 \%$ \\
\hline
\end{tabular}

Table 1: The effect of sex on graft survival (Busson/Benoit 1997).

patients on the waiting list were of blood type 0 while $40 \%$ of all donors had this blood type. Using a frequency of $50 \%$ for organs and patients of blood type $\mathrm{O}$, the uppermost curve labelled 'alpha $=1$ ' in Figure 3 shows the probability of misallocating an organ of blood type $\mathrm{O}$ to a patient of a different blood type. After only a comparatively small number of 800 allocations, the probability of misallocating drops below $1 \%$.

It has been argued that additional non-immunological factors influence graft survival rates. The conclusions of Busson/Benoit (1997) would imply that the current allocation process disadvantages men. According to this study, men maximize their graft survival rates when they receive an organ from a male donor, while the influence of the donor's sex is much less pronounced for female recipients (cf. Table 1). The authors find that a sex mismatch erodes graft survival rates at 3 years for male recipients by about $6 \%$. Persijn/Smits/De Meester (1999) also report an effect in this direction but their finding is less pronounced. Meier-Kriesche et al. (2001) find no such effect but they seem not to differentiate between patients receiving an organ of a different sex. Doubtlessly, more studies are needed to resolve these questions. We can nonetheless note that matching for these non-immunological criteria opens a Pandora's box of moral dilemmas under the current allocation methods. We study the example of men matching for sex. AWT again offers a clear improvement over the status quo for the disadvantaged sex and reduces any imbalances that could have been avoided. In 1999, women occurred in the European cadaveric kidney donor population roughly with a frequency of $43 \%$. For the sake of brevity, we approximate this situation by consulting Figure 3 for a frequency of $50 \%$. A misallocation would occur if we allocated a male organ to a female patient while there was a shortage of male organs among male patients with the same waiting time as the female patient. The figure shows that the probability of such a misallocation quickly falls to zero. This is true even if only some proportion $\alpha$ of all men decide to be selective and match the donor organ for sex.

\subsection{Medical Optimality}

AWT resolves the moral dilemma of reconciling the maximization of an individual's well-being with a societal notion of distributive justice. As more nonimmunological and morally sensitive factors emerge as predictors of graft survival rates, the moral properties of AWT have implications for the medical optimality of our proposal. AWT allows criteria like sex, age, race or any other additional criteria to be taken into account without violating moral fairness norms. This 
procedure therefore produces outcomes that are as close to the medical optimum as current medical knowledge allows and as close as the patients desire. If AWT falls short of the medical optimum, it does so in response to how the patients value the importance of graft survival rates vis-a-vis waiting time. There are no objective grounds for criticizing the patients' choice.

Patients have a definite incentive to maximize their graft survival rates, especially in view of medical side-effects and complications arising from poor medical compatibility. Moreover, their choice will be guided by medical experts and we will retain some control over the range of the choice that can be offered to the patients on medical grounds. Patients in an overall poor medical condition may, for instance, be suspended from the waiting list until their condition improves. This suggests that the medical performance of AWT may not be far from the medical optimum. It may in principle even perform better than the current allocation mechanisms that do not take non-immunological criteria into account. If the current allocation rules were in the future to include non-immunological criteria, they would become entangled in moral dilemmas concerning the maximization of individuals' expected medical outcome and norms of distributive justice. Wujciak/Opelz (1993) reach an opposing conclusion in their discussion of a similar 'first in, first out' allocation method. The poor performance of this method in Wujciak and Opelz's simulation depends on the fact that an organ is allocated to the first patient on the waiting list without considering the patient's own choice. In particular, the patient is not given a choice between accepting or declining the allocated kidney on the basis of her preferences for waiting time, HLA matching, PRA sensitization etc. Their simulation therefore assumes that organs are randomly accepted and rejected by patients regardless of their preferences. This is an unrealistic assumption since patients have a very clear incentive to choose organs promising a high survival rate.

AWT could widen the acceptability of age-specific matching that is, for example, intended by Eurotransplant's 'old-for-old' programme. Such programmes become superfluous because waiting time creates an incentive for older people to consider accepting an organ from an older patient without a great loss in their graft survival rate. AWT also allows an increased use of marginal organs based on circumspect local decision. The patients' informed consent avoids severe legal problems of adequate disclosure of medical risks (for the USA, cf. Canterbury v Spence, Ref. 464F dd 772, 1972, USCA District of Columbia; for Great Britain, cf. Bolam, 2 All ER118, 1957).

\subsection{Interpersonal Comparisons}

The properties of allocation methods have been studied extensively by a large body of literature in social choice theory (Mongin/d'Aspremont 1998). We here present a positive conclusion from this literature supporting our proposal. The current Eurotransplant and UNOS allocation rules are special cases of a general class of rules whose properties are very well understood. ${ }^{5}$ The most crucial insight of the last 30 years of theoretical research on allocation rules concerns the

\footnotetext{
5 Rules maximizing the sum of some point scheme are known as utilitarian rules.
} 
critical importance of trade-offs between the well-being of different individuals. Such trade-offs require not only intra-personal comparisons of a single individual's well-being under different allocations, but also inter-personal comparisons of different individuals' well-being under different allocations. The following statements provides an example of such comparisons.

Intra-personal comparison: Patient 1 is better off with 1 HLA-mismatch and 6 years of waiting than with 3 HLA-mismatches and 3 years of waiting.

Inter-personal comparison: $1 \mathrm{HLA}-$ mismatch and 6 years of waiting for patient 1 is better than 3 HLA-mismatches and 3 years of waiting for patient 2 .

The allocation rules used by Eurotransplant and UNOS make both intra- and inter-personal comparisons that are implicit in the scheme through which patients are awarded points. The point scheme implicitly determines by how much patient 1's well-being with 1 HLA-mismatch and 6 years of waiting outweighs patient 2's well-being with 3 HLA-mismatches and 3 years of waiting. These interpersonal comparisons may not be intentional. At least in the published literature, these point schemes are judged by equity criteria pertaining to the overall allocation of organs and not to trade-offs among individuals. Wujciak/Opelz 1993, for example, point out that their XCOMB point scheme yields overall survival rates that are close to the optimum (using only HLA matching as a predictor of medical outcome). Leffell/Zachary 1999 evaluate the updated 1995 UNOS scheme by examining whether they promote racial equality and related criteria. De Meester et al. (1999) explicitly evaluate the Eurotransplant rule on the basis of weighing the societal aims of medical efficiency and equity. The considerations of the preceding sections show that AWT agrees with those implicit interpersonal comparisons that derive from the more fundamental motivation to protect rare or otherwise disadvantaged patient types. After only a few organ allocations, the probability becomes small that an organ is misallocated to a patient of an advantaged type to the detriment of a disadvantaged patient. Even if unintended, more widespread interpersonal comparisons are implicit in the Eurotransplant and UNOS allocation rules and directly affect the patient's eligibility and opportunity.

There is no moral basis for making these comparisons. It is both practically impossible and morally inappropriate to make intra- and inter-personal comparisons of well-being on behalf of the patients. Firstly, these comparisons would require a quantitative evaluation of the effect of additional waiting time. A particular quantification often turns out to be the artifact of a particular elicitation method (cf. the difference between the studies in this volume). Secondly, only patients have access to highly relevant information about their own situation, such as the physical, psychological and emotional suffering that additional waiting time inflicts on themselves and their families at a given point in time. Thirdly, even medical experts disagree on how HLA matches and waiting time should be weighted. No satisfactory method has ever been proposed for settling such disagreements about the relative weight of medical and non-medical criteria. For the purpose of policy recommendations, all three of these problems severely limit 
the usefulness of studies that elicit medical expert's subjective trade-offs. Apart from doubts about the feasibility of making interpersonal comparisons systematically and coherently, it is also morally inappropriate for society to make such decisions on behalf of a patient. This is especially true if non-medical considerations about the patient's particular psychological, social or emotional situation are permitted. Compromising expected graft survival time in favour of a shorter waiting period or other non-medical aspects is a deeply personal choice that should not be taken away ifrom the patient. As so many personal choices, it is also an extremely complex and difficult choice for which the patient must be offered any possible support.

This realization is the first step of a positive and constructive argument. Luce/Raiffa (1957) point out that an inability to make inter-personal comparisons commits us to a particular class of allocation rules. AWT falls within this distinguished class of allocation rules. In other words, there are strong additional reasons in favour of AWT over and above the optimality properties that we have established above.

Theorem. If we cannot make any interpersonal comparisons and if we want Pareto optimality, then we must define some priority ranking among individuals and then let the individuals determine the allocation in the order of their seniority. That is, we must choose an allocation that maximizes the welfare of the individual with the highest priority, then that of the individual with the second highest priority etc.

For a detailed proof, cf. Gevers (1979). Although this theorem does not tell us how to prioritize patients, it tells us that AWT is of the correct type. We will now discuss what factors other than waiting time should determine priority.

\subsection{Special Considerations}

We now turn to group of patients that seem to merit special consideration by an allocation mechanism. While for most patients the allocation of a donor kidney is mainly an issue for their mid-term quality of life, some patients are threatened by severe impairments of their future health and their future ability to enjoy a good quality of life if they do not quickly receive a transplant. Children, for instance, seem to deserve special consideration in the allocation process because their physical development during adolescence is severely impaired without a transplant. For patients of high medical urgency, a delayed offer of a transplant can even result in death, while the overall mortality rate of patients on the waiting list is comparatively low. Children and patients of high medical urgency therefore simply do not have the time to wait for their entitlement under AWT or any other allocation rule. It seems to be agreed that such patients must be moved forward on the waiting list. By assigning bonus waiting times to such patients, we can determine whether they should be moved to the very front of the waiting line or to some region close to the front.

By analogous means, we can adjust for regional and international importexport imbalances. This is a problem of particular political importance for Eurotransplant where different nationalities contribute to and receive organs from 
a shared pool of donor organs, thus increasing the probability of finding good HLA matches. AWT treats patients of different origin equally and any imbalances of imports and export are the result of these patients' free choices. There may however exist some disadvantages for which certain regions or nationalities deserve compensation. Since, for instance, cold ischemia time is an important predictor of graft survival rates, patients from countries on the periphery of the common allocation area are at a disadvantage. Caused by morally irrelevant topographical facts, they have to accept on average somewhat longer transport times and longer cold ischemia times. In order to offset such effects, we can again award a bonus waiting time to patients from the periphery and thus move them ahead on the waiting list.

More generally, there is a very simple algorithm for equalizing imbalances in attributes that for moral reasons should be irrelevant. Think of attribute $A$ as a central geographical region and of attribute $B$ as a peripheral geographical region in the allocation area. ${ }^{6}$ A general method of compensating for irrelevant attributes calculates on a regular basis the average waiting times $\bar{W}_{A}$ and $\bar{W}_{B}$ of patients of type $A$ and type $B$. If $\bar{W}_{B}$ exceeds $\bar{W}_{A}$, we award patients of type $B$ the difference $\bar{W}_{B}-\bar{W}_{A}$ as their bonus waiting time. This method assumes that the distribution of time preferences is identical among patients from area $A$ and area $B$. If patients from area $B$ were on average less concerned about long waiting times and more about good HLA matches, then this imbalance would be the result of a free choice of the $B$ patients. The question of which attributes deserve compensation needs to be studied in more detail.

In the past, there has been a persistent shortage of donor organs and an excess of patients in need of a graft. The European data for 1999 show that the net inflow into the waiting list exceeded the net outflow by 198 patients even if we hypothetically exclude additional demand through retransplantations. ${ }^{7}$ If this trend continues, average waiting time will increase by 0.78 months per year, or about 4 months in 5 years. Under these conditions, future patients are disadvantaged relative to current patients (leaving aside potential but yet unforeseen advances in medical technology). We might consider awarding patients who join the waiting list at a later time a bonus waiting time of 0.78 months per year to offset the birth-time disadvantage of future patients. This might be felt to restore injustices arising from the morally arbitrary hour of a person's birth but, of course, cannot combat the dearth of donor kidney.

The same reasoning could be applied to the distribution of kidneys among small and large transplantation centres. We could by the same method award a bonus to patients from small transplantation centres if the strengthening of

${ }^{6}$ The attributes $A$ and $B$ must be such that the patients' choices do not depend directly on $A$ and $B$. If, for example, all Austrian patients (attribute $A$ ) had a preference for Belgian kidneys, then Austrian patients would clearly have to wait much longer than any other patient group (attribute $B$ ). This increased waiting time is, however, the result of a free choice of the Austrian patients.

7 If we allow patients to rejoin the waiting list after they have lost their first transplant, the inflow rate into the waiting list increases even more and further disadvantages future patients. This poses additional questions about how re-transplant patients should be treated in the allocation process. 
small transplantation centres is politically desired. The moral relevance of some other attributes is much harder to evaluate. Should the patient, e.g. a diabetic smoker, be held responsible for his past behaviour leading to kidney failure? Such attributes play currently no role.

\subsection{Practicability}

The decision to accept or to reject a donor organ for transplantation poses a complex problem that requires a difficult final decision by the patient. Tragically, most important decisions in life are of this nature. The patients in medical care will benefit from the counsel of their medical advisers and the decision-making process must be structured so as to minimize the psychological discomfort or anxiety experienced by the patient. To relieve the stress of an instantaneous decision and to expedite the allocation process, the patients are asked to draft, together with their medical advisers, a plan for when to accept an organ of what type. The patient has the option to delegate the decision to the transplant surgeon or to rely on the recommendations of a panel of medical experts such as the commission that produces the European Best Practice Guide on Renal Transplantation. Patients with impaired mental abilities or with mental disorders are represented by their guardians or are treated under a default plan devised by an expert panel. The patients' choices can evolve and consolidate over time since they have the opportunity to revise their stated preferences on a regular basis. A project is currently under way to develop a cognitively and emotionally tractable format in which patients can absorb medical information about their situation.

\section{Conclusion}

We summarize the properties of our proposal. Our proposal resolves concerns about distributive justice:

- Disadvantaged patient types are automatically protected.

- Morally sensitive attributes such as sex, age and race can be used as selection criteria without creating injustices.

- Age-specific programmes, such as Europe's old-for-old programme, become superfluous. Waiting time creates an incentive for older people to consider accepting an organ from an older patient without a great loss in their graft survival rate.

Patients and their medical advisers locally determine trade-offs between immunological and non-immunological criteria and between medical and nonmedical criteria:

- The patients' informed consent allows an increased use of marginal donor organs and protects transplant centres from liability claims. 
- Involving patients in decision making has positive psychological effects and may be conjectured to increase post-operative compliance with the medication regime.

- Prioritization by waiting time is an extremely transparent and easy to understand criterion. Point schemes, on the other hand, impose a regime of abstract calculations that patients, physicians and politicians might find more difficult to accept.

Our allocation method adapts flexibly to new medical developments:

- The allocation mechanism remains impartial in the controversy about the relative importance of HLA mismatching and cold ischemia times.

- No revisions of the mechanism are needed when new medical factors emerge or when of new medical technology is created.

A crucial prerequisite of AWT is the development of a support system for patients and their surgeons. Central authorities such as UNOS or Eurotransplant will continue to play a crucial role by formulating best practice guidelines, by monitoring the information and the support that is provided to the patients and by maintaining the allocation process.

\section{Appendix: Statistical Analysis}

Any allocation algorithm has to operate in a stochastic environment in which organs and patients of different types become available in a random order and at random times. We wish to find the probability that a patient of a 'disadvantaged' type is passed over in favour of a patient of an 'advantaged' type. We derive this probability under the worst-case assumptions that advantaged patients can accept any organ and disadvantaged patients can only accept organs of their own type. We are at this point not interested in how patients discriminate between subtypes of organs. It is then enough that we distinguish two medical types $(1,2)$ of patients and organs. For patients of type 2, we also distinguish between two sub-types $2 a, 2 b$. We adopt the following worst-case rules that will govern the dynamics of the waiting list:

1. Patients of type 1 and type $2 b$ accept organs of type 1 and type 2 .

2. Patients of type $2 a$ accept only organs of type 2 .

The probability that an organ of type 1 becomes available is $1-q$ and the probability that an organ of type 2 becomes available is $q$. The probability that a patient of type 1 is added to the waiting list is $1-p$, the probability that a patient of type $2 a$ is added to the waiting list is $\alpha p$ and the probability that a patient of type $2 b$ is added to the waiting list is $(1-\alpha) p$. We assume that drop-out rates from the waiting list have already been subtracted from the rates with which patients join the waiting list. We assume that these probabilities 
are independent of each other. In other words, the event of an organ becoming available does not affect the probability of a patient with a certain type joining the waiting list. We also assume that these probabilities remain constant over time and that patients do not rejoin the waiting list for a second transplant. We assume that the waiting list is long enough so that there is always demand for an organ. In the future this situation may perhaps change, but it is the reality of today. Thanks to this simplifying assumption, we are able to treat the problem with analytical means. We model the waiting list by a sequence $x_{1}, x_{2}, \ldots, x_{n}, \ldots$ where $x_{n}=1,2 a, 2 b$ and where $x_{1}$ is the type of the patient who has been waiting the longest. Before any organs have been allocated, the probability that $x_{n}=1,2 a, 2 b$ is $1-p, \alpha p$ and $(1-\alpha) p$, respectively. The composition of the waiting list changes with time depending on the allocation rule and depending on the organs that become available.

In an optimal allocation for the selective type $2 a$, any patient of this type receives an organ of type 2 so that a proportion of $q-\alpha p$ type 2 organs that remains for the patients of all other types. If this remaining portion of type 2 organs is allocated randomly among the patients of type 1 and type $2 b$, then the smallest possible probability of allocating a type 2 organ to a patient of type 1 is

$$
\begin{array}{lll}
p_{\min }=(q-\alpha p) \cdot \frac{1-p}{1-\alpha p}, & \text { when } \alpha p \neq 1 . \\
p_{\min }=0, & \text { else. } &
\end{array}
$$

We derive the probability $p_{n}$ that an organ of type 2 is allocated to a patient of type 1. Such an allocation occurs when an organ of type 2 arrives while a patient of type 1 is at the first position of the waiting list after the $n-1$ st arrival of an organ. Recall that patients of type 1 and type $2 b$ accept any organ. Hence, if we consider a patient of one of these types at position $k$, we know that no patient with a rank $l \geq k$ has so far received an organ. A patient at rank $l$ after $n$ organ arrivals must therefore have held rank $l+n$ before any organs arrived. Hence, the probability of the patient at rank $l$ being of type $i$ after $n$ organ arrivals is identical to the probability that, before any organs have arrived, the patient at rank $l+n$ is of type $i$. If we know that the initial part of the sequence of patients after $n$ organ arrivals is $\langle\vec{x}, 1\rangle$ or $\langle\vec{x}, 2 b\rangle$, then the probability of the sequence continuing as $\vec{y}$ is only determined by the number $m_{1}$ of patients of type 1 in $\vec{y}$, the number $m_{2}$ of patients of type $2 a$ in $\vec{y}$ and the number $m_{3}$ of patients of type $2 b$ in $\vec{y}$. For $i=1,2 b$,

$$
P_{n}(\langle\vec{x}, i, \vec{y}\rangle \mid\langle\vec{x}, i\rangle)=(1-p)^{m_{1}}(\alpha p)^{m_{2}}(p-\alpha p)^{m_{3}}
$$

When $\alpha p \neq 1$, we also have

$$
P_{n}(\langle\vec{x}, 1\rangle \mid\langle\vec{x}, 1\rangle \text { or }\langle\vec{x}, 2 b\rangle)=\frac{1-p}{1-\alpha p}
$$

For any $n \in \mathbb{N}$ and $k \in \mathbb{N}^{+}$, we write $Q_{n}(k)$ for the probability that the first patient of type 1 or type $2 b$ occurs at position $k$ after the $n$th arrival of an organ. For any $k \in \mathbb{N}^{+}$, we have 


$$
Q_{0}(k)=(\alpha p)^{k-1}(1-\alpha p)
$$

Suppose we already know $Q_{n}(k)$ (for any $k \in \mathbb{N}$ ). We now derive $Q_{n+1}(k)$ (for any $k \in \mathbb{N}$ ). In a $(n+1)$-sequence of organs, the last organ is of type 1 (Case 1 ) with probability $1-q$ and of type 2 (Case 2 ) with probability $q$. We consider all $l \in \mathbb{N}^{+}$sub-cases in which the first patient of type 1 or type $2 b$ occurs at position $l$. The probability $Q_{n}(l)$ of each such sub-case is independent of the type of the $n+1$ st organ and thus independent of Case 1 and Case 2. Case 1: The $n+1$ st organ is of type 1 . Such an organ is rejected by patients of type $2 a$ and accepted by patients of type 1 and type $2 b$. Conditional on $l \leq k$, the probability that, after $n+1$ organ arrivals, the first patient of type 1 or type $2 b$ occurs at rank $k$ is (by (2)) the probability of $l$ being followed by $k-l$ patients of type $2 a$ and 1 patient of type 1 or type $2 b$, i.e., $(\alpha p)^{k-l}(1-\alpha p)$. Conditional on $l>k$, this probability is zero. Case 2: The $n+1$ st organ is of type 2. Such an organ is accepted by any patient. Conditional on $l=1$, the probability that, after $n+1$ organ arrivals, the first patient of type 1 or type $2 b$ occurs at rank $k$ is (by (2)) the probability of the first patient being followed by $k-1$ patients of type $2 a$ and 1 patient of type 1 or type $2 b$, i.e., $(\alpha p)^{k-1}(1-\alpha p)$. Conditional on $l=k+1$, the probability that, after $n+1$ organ arrivals, the first patient of type 1 or type $2 b$ occurs at rank $k$ is trivially one. Conditional on $2 \leq l \leq k$ or $k+2 \leq l$, this probability is zero. We thus obtain

$$
\begin{aligned}
Q_{n+1}(k) & =(1-q)\left(\sum_{1 \leq l \leq k} Q_{n}(l) \cdot(\alpha p)^{k-l}(1-\alpha p)\right) \\
& +q\left(Q_{n}(1) \cdot(\alpha p)^{k-1}(1-\alpha p)+Q_{n}(k+1)\right)
\end{aligned}
$$

Moreover, we can now derive the probability that a patient of type 1 is at the front of the waiting list after $n \in \mathbb{N}$ organ arrivals. When $\alpha p=1$, this probability is zero and $Q_{n}(1) \cdot \frac{1-p}{1-\alpha p}$ otherwise (by (3)). We finally arrive at the probability $p_{n}$ that the $n$th organ $\left(n \in \mathbb{N}^{+}\right)$is misallocated.

$$
\begin{array}{lll}
p_{n}=Q_{n-1}(1) \cdot q \frac{1-p}{1-\alpha p}, & \text { when } \alpha p \neq 1 \\
p_{n}=0, & \text { else. }
\end{array}
$$

Next, we obtain the expected proportion of losses of organs of type 2 after $n \in \mathbb{N}^{+}$ organ arrivals:

$$
\begin{array}{ll}
e_{n}=\frac{E_{n}}{q \cdot n}, & \text { when } q \neq 0 . \\
e_{n}=0, & \text { else. }
\end{array}
$$




\section{Bibliography}

Busson, M./G. Benoit (1997), Is Matching For Sex and Age Beneficial to Kidney Graft Survival?, in: Clinical Transplantation 11, 15-18

Foley, D. (1967), Resource Allocation and the Public Sector, in: Yale Economic Essays 7, 45-98

Gevers, L. (1979), On Interpersonal Comparability and Social Welfare Orderings, in: Econometrica 47, 75-89

Hild, M. (2001a), Stable Aggregation of Preferences, in: California Institute of Technology, Social Science Working Paper 1112, available at www.hild.org

- (2001b), Policy Reversals in Risk Management: The Effect of Refined Analyses, in: California Institute of Technology, Social Science Working Paper 1116, available at www.hild.org

Leffell, M. S./A. A. Zachary (1999), The National Impact of the 1995 Changes to the UNOS Renal Allocation System, in: Clinical Transplantation, 13, 287-295

Luce, R. D./H. Raiffa (1957), Games and Decisions, New York

De Meester, J./G. G. Persijn/J. Smits/Y. Vanrenterghem (1999), The New Eurotransplant Kidney Allocation System: A Justified Balance between Equity and Utility?, in: Transplant International 12, 299-300

Meier-Kriesche, H.-U/A. O. Ojo/S. F. Leavey/A. B. Leichtman/J. C. Magee (2001), Gender Differences in the Risk for Chonic Renal Allograft Failure, in: Transplantation 71, 429-432

Mongin, P./C. d'Aspremont (1998), Utility Theory and Ethics, in: S. Barbera/P. Hammond/C. Seidl (eds.), Handbook of Utility Theory, chapter 10, pages 371-481, Dordrecht

Persijn, G. G./J. Smits/J. De Meester (1999), Nonimmunologic Matching in Renal Transplantation, in: Transplantation Proceedings 31, 1772-1774

Thomson, W./H. R. Varian (1985), Theories of Justice Based on Symmetry, in: L. Hurwicz/D. Schmeidler/H. Sonnenschein (eds.), Social Goods and Social Organizations: Essays in Memory of Elisha Parzner, 107-129, Cambridge

Tinbergen, J. (1953), Redeljke Inkomensverdeling, Haarlem

Wujciak, T./G. Opelz (1993), Computer Analysis of Cadaver Kidney Allocation Procedures, in: Transplantation 55, 516-521 